Research Article

\title{
The Effects of Age and Reproduction on the Lipidome of Caenorhabditis elegans
}

\author{
Qin-Li Wan, ${ }^{1}$ Zhong-Lin Yang, ${ }^{2,3}$ Xiao-Gang Zhou, ${ }^{1}$ Ai-Jun Ding, ${ }^{1}$ Yuan-Zhu Pu, ${ }^{1}$ \\ Huai-Rong Luo $(1),{ }^{1,2}$ and Gui-Sheng $\mathrm{Wu}(\mathbb{1})^{1}$ \\ ${ }^{1}$ Key Laboratory for Aging and Regenerative Medicine, Department of Pharmacology, School of Pharmacy, \\ Southwest Medical University, Luzhou, Sichuan 646000, China \\ ${ }^{2}$ State Key Laboratory of Phytochemistry and Plant Resources in West China, Yunnan Key Laboratory of Natural \\ Medicinal Chemistry, Kunming Institute of Botany, Chinese Academy of Sciences, Kunming, Yunnan 650201, China \\ ${ }^{3}$ University of Chinese Academy of Sciences, Beijing 100039, China
}

Correspondence should be addressed to Gui-Sheng Wu; wgs@swmu.edu.cn

Received 30 December 2018; Accepted 25 February 2019; Published 9 May 2019

Academic Editor: Consuelo Borrás

Copyright (c) 2019 Qin-Li Wan et al. This is an open access article distributed under the Creative Commons Attribution License, which permits unrestricted use, distribution, and reproduction in any medium, provided the original work is properly cited.

\begin{abstract}
Aging is a complex life process, and a unified view is that metabolism plays key roles in all biological processes. Here, we determined the lipidomic profile of Caenorhabditis elegans (C. elegans) using ultraperformance liquid chromatography high-resolution mass spectrometry (UPLC-HRMS). Using a nontargeted approach, we detected approximately 3000 species. Analysis of the lipid metabolic profiles at young adult and ten-day-old ages among wild-type N2, glp-1 defective mutant, and double mutant daf$16 ; g l p-1$ uncovered significant age-related differences in the total amount of phosphatidylcholines (PC), sphingomyelins (SM), ceramides (Cer), diglycerides (DG), and triglycerides (TG). In addition, the age-associated lipid profiles were characterized by ratio of polyunsaturated (PUFA) over monounsaturated (MUFA) lipid species. Lipid metabolism modulation plays an important role in reproduction-regulated aging; to identify the variations of lipid metabolites during germ line loss-induced longevity, we investigated the lipidomic profiles of long-lived $g l p-1 /$ notch receptor mutants, which have reproductive deficiency when grown at nonpermissive temperature. The results showed that there was some age-related lipid variation, including TG 40:2, TG 40:1, and TG 41:1, which contributed to the long-life phenotype. The longevity of $g l p-1$ mutant was daf-16-dependent; the lipidome analysis of $d a f-16 ; g l p-1$ double mutant revealed that the changes of some metabolites in the $g l p-1$ mutant were daf16-dependent, while other metabolites displayed more complex epistatic patterns. We first conducted a comprehensive lipidome analysis to provide novel insights into the relationships between longevity and lipid metabolism regulated by germ line signals in C. elegans.
\end{abstract}

\section{Introduction}

Life expectancy in humans has dramatically increased worldwide [1]. Aging is often accompanied by diseases and disabilities, including cardiovascular diseases, neurodegenerative diseases, infectious diseases, and cancers $[2,3]$. Thus, understanding the physiological characteristics of aging is of extreme importance to enable populations to grow old in good health and without disease.

Several pathways involved in aging mechanisms (e.g., mitochondrial health, DNA damage, proteostasis imbalance, systemic inflammation, epigenetic modifications, and nutrient-sensing pathways) have been associated with undesirable metabolic alterations $[4,5]$. Without exception, metabolism changes also occur during reproductionregulated aging. Using comprehensive nontargeted metabolomics, we previously demonstrated that aging is a process of metabolome remodeling and that long-lived germ lineless $g l p-1$ mutants regulated the levels of many age-variant metabolites to attenuate aging, including pyrimidine metabolism, purine metabolism, citric acid cycle, glycerophospholipid metabolism, and starch and sucrose metabolism [6]. These findings elucidated the mechanism of reproductionregulated aging based on the metabolism of small molecules 
of endogenous water. Undoubtedly, the metabolism of nonwater-soluble molecules is also involved in reproductionregulated aging.

Previous studies have reported that sacrificing fertility to induce lifespan extension is evolutionarily conserved $[7,8]$. In C. elegans, germ line removal not only lengthens lifespan but also increases fat accumulation, as observed using lipidlabeling dyes and gas chromatography (GC) $[9,10]$. Notably, a reproductive deficiency leading to enhanced fat accumulation has been observed in many other organisms, including both invertebrates (e.g., drosophila) [11, 12] and vertebrates (e.g., mice, rats, and monkeys) [13-15].

In C. elegans, a RNA-Seq study demonstrated that upon GSC removal the genes associated with lipid metabolism were upregulated by DAF-16/FOXO3A and/or TCER1/TCERG1 [16]. These genes included enzymes that initiate de novo fatty acid synthesis (including pod-2 (encodes acetyl CoA carboxylase, ACC), mlcd-1 (malonyl CoA decarboxylase 1, MLCD), and fasn-1 (encodes fatty-acid synthase, FAS)) and the genes encoding diacylglycerol acyltransferase (DGAT) enzymes, which catalyze the final step in triglyceride (TAG) production (including dgat-2, acs-22, mboa-2, Y53G8B.2, and K07B1.4). In addition, in $g l p-1$ mutants, expression of desaturase enzymes, which catalyze the conversion of saturated fatty acids (SFAs) into unsaturated fatty acids (UFAs), was enhanced in a NHR-49/PPAR $\alpha$ - and NHR-80/HNF4-dependent manner, thereby increasing the monounsaturated fatty acid MUFA and reducing SFA levels. Furthermore, expression of fat- 6 and fat-7, encoding stearoyl-CoA 9-desaturase (SCD) enzymes, which transform stearic acid (SA, a SFA) to oleic acid (OA, a MUFA), was obviously altered in $g l p-1$ mutants. Additionally, in $g l p-1$ mutants, expression of genes involved in $\beta$-oxidation was upregulated in an NHR-49/PPAR $\alpha$-dependent manner [17]. Moreover, following GSC loss, upregulation of the expression of the genes encoding lipase and lipase-like protein (i.e., lipl-4, orthologous to the human lipase LIPA) was mediated by DAF-16/FOXO3A, TCER-1/TCERG1, or SKN-1/NRF2 $[16,18,19]$. These studies illustrated that in germ line loss animals, lipid catabolism and anabolism are simultaneously elevated, and germ line loss animals show fat accumulation phenotypes. Expectedly, in $g l p-1$ mutants, lipid staining and gas chromatography/mass spectrometry (GC/MS) analyses displayed increased levels of TAG and some fatty acids, including C 16:0, C 16:1n7, C 18:0, C $18: 1 n 7$, and C 18:3 $[9,10,16]$. However, these studies only highlighted changes in the levels of overall lipids and fatty acids in $g l p-1$ mutants, barely elucidating the role of individual intact lipid molecules in the regulation of aging by reproduction. Therefore, the aim of the present study was to clarify the role of lipid metabolism in the regulation of aging by reproduction based on lipidomics to obtain intact lipid information and lipid metabolic profiles.

Lipidomics, a branch of metabolomics, refers to the characterization and analysis of the lipid complement of biological systems. Lipidomics can be achieved through comprehensive measurement of the lipid metabolic profiles based on analytical chemistry principles and technological tools, particularly mass spectrometry $[20,21]$. In recent years, reflecting the development of MS, lipidomic technology has been widely used in many fields, such as organic biofluids with environmental, pathological, or toxicological stress [2225]. Furthermore, the lipidome has been used in aging studies. For example, the Leiden Longevity Study demonstrated that specific lipids are associated with familial longevity [26]. Additional lipidomic analyses revealed that some lipids containing phosphatidylethanolamines, phosphatidylcholines, and sphingomyelins are associated with aging in humans [27]. Other studies have revealed that phospho/sphingolipids may represent putative markers and biological modulators of healthy aging in humans [28]. Furthermore, studies have illustrated that $\mathrm{PC}(\mathrm{O}-34: 1)$ and $\mathrm{PC}(\mathrm{O}-34: 3)$ are positively associated with longevity and negatively associated with diabetes $[29,30]$.

The objectives of the present study were to characterize the lipid metabolic profiles of C. elegans hermaphrodites during aging and identify germ line signals that regulate aging. Here, we assessed the lipid metabolic phenotype of whole C. elegans between two physiological ages among different mutants (e.g., wild type, $g l p-1$ mutants, and daf-16; $g l p-1$ double mutants) using ultraperformance liquid chromatography high-resolution mass spectrometry (UPLC-HRMS), with multivariate statistics analysis, including unsupervised principal component analysis (PCA) as well as hierarchical and supervised orthogonal projection to latent structure with discriminant analysis (OPLS-DA). These results demonstrated that reproductive signals affect lifespan by regulating metabolic changes, and some of these metabolic controls were mediated by FOXO/DAF-16.

\section{Materials and Methods}

2.1. Culture of Nematodes. Wild-type N2, CF1903 glp1(e2144)III, and CF1880 daf-16(mu86)I;glp-1(e2144)III were obtained from the Caenorhabditis Genetics Center (CGC) and maintained under standard conditions on nematode growth media (NGM) with Escherichia coli OP50 as previously described, unless otherwise stated [31].

2.2. Sample Preparation for Metabolomic Analysis. The strains were cultured for 2-3 generations prior to collection. To eliminate germ cells of the $g l p-1(e 2144)$ and daf16(mu86);glp-1(e2144) alleles, all strains were synchronized and incubated at $20^{\circ} \mathrm{C}$ for $12 \mathrm{~h}$, shifted to $25^{\circ} \mathrm{C}$, grown to the L4 stage, and subsequently shifted back to $20^{\circ} \mathrm{C}$, followed by harvest. The samples were prepared as previously described [6]. Briefly, a large sample of young adult (YA) or day-10 adult $(10 \mathrm{~A})$ worms $(\sim 8000)$ was pooled and washed with M9 buffer and subsequently collected. All samples were snap frozen in liquid nitrogen and dried overnight in vacuo at a low temperature, weighed, and stored at $-80^{\circ} \mathrm{C}$ until extraction. For samples of old worms, $10 \mu \mathrm{M} 5$-fluoro- $2^{\prime}$-deoxyuridine (FUdR, Sigma) was used to prevent selffertilization. It is known that FUdR influences metabolism of worms [32], but the biomass of worms required for lipidomic analysis cannot be collected without using FUdR or a similar intervention. While in order to reduce the influence 
of FUdR, all strains were maintained on the same condition, as previously described [6].

Lipid metabolites from C. elegans samples were extracted three times with $600 \mu \mathrm{L}$ of precooled $\mathrm{CH}_{2} \mathrm{Cl}_{2} / \mathrm{MeOH}(2: 1)$ using a TissueLyser at $55 \mathrm{~Hz}$ for $90 \mathrm{~s}$. All extracts were subjected to centrifugation ( $12000 \mathrm{rpm}$ for $10 \mathrm{~min}$ at $4^{\circ} \mathrm{C}$ ), subsequently stored at $-80^{\circ} \mathrm{C}$ until further analysis.

\subsection{Lipidomic Analysis Using UPLC-HRMS}

2.3.1. Sample Preparation. The samples were thawed at room temperature, centrifuged at $12,000 \mathrm{rpm}$ for $10 \mathrm{~min}$ at $4^{\circ} \mathrm{C}$, and analyzed using UPLC-HRMS.

2.3.2. Apparatus and Analytical Conditions. According to Witting et al. [33], liquid chromatography was performed using a reversed-phase $\mathrm{C} 18$ column (CORTECS UPLC C18, waters, $1.6 \mu \mathrm{m} 150 \times 2.1 \mathrm{~mm}$ diameter column) with a flow rate of $300 \mu \mathrm{L} / \mathrm{min}$ at $35^{\circ} \mathrm{C}$, and $5 \mu \mathrm{L}$ of sample was injected. The mobile phase comprised ACN/H2O $(3: 2, v / v)(\mathrm{A})$ and iPrOH/ACN $(9: 1, v / v)(\mathrm{B})$, and both $\mathrm{A}$ and $\mathrm{B}$ contained $0.1 \%$ formic acid and $5 \mathrm{mM}$ ammonium formate. The initial eluent comprised $32 \%$ solvent $B$, which was held for 2 min; the percent of buffer B was then gradually increased to $97 \%$ in $30 \mathrm{~min}$, was held for $5 \mathrm{~min}$, and was then returned to the initial condition in $0.1 \mathrm{~min}$. The column was reequilibrated for $4.9 \mathrm{~min}$, and the total run time was $40 \mathrm{~min}$.

Analyses were conducted using the Agilent 1290 UPLC System (Agilent, Santa Clara, CA) connected to an Agilent 6500 Q-TOF Mass Spectrometer (Agilent, Santa Clara, CA). The parameter of mass spectrometry analyses in positive ion mode was set previously described, with some modifications [6].

2.3.3. Data Processing. Raw files from UPLC-MS were converted to the mzData format using Masshunter Qualitative Software (Agilent, Santa Clara, CA). Peak detection, alignment, and integration were performed using the opensource software XCMS and CAMERA implemented with the freely available $\mathrm{R}$ statistical language (version 3.2.2). The procedures and parameters were conducted according to previous studies, with some modifications [34]. Identification of metabolites was performed based on their molecular ion masses and $\mathrm{MS}^{\mathrm{n}}$ fragmentation compared with the literature and metabolomic library entries of purified standards, such as LIPID MAPS [35], LIPID BANK [36], the HMDB [37], Lipidhome [38], and LIPIDAT [39]. Subsequently, the putative identifications were verified through comparisons of the retention time matches to those of authentic standard compounds. In addition, we also purchased some commercially available purified standard compounds from Avanti Polar Lipids (Alabaster, AL, USA) to identify metabolites. These standard compounds include heptadecasphing-4enine, heptadecasphinganine, C17 Sphingosine-1-phosphate, C17 Sphinganine-1-phosphate, $\quad \mathrm{SM}(\mathrm{d} 18: 1 / 12: 0)$, Cer(d18:1/12:0), CerP(d18:1/12:0), Cer(d18:1/25:0), d5DG(14:0/0:0/14:0), d5-DG(15:0/0:0/15:0), d5-DG(16:0/0:0/16:0), d5-DG(17:0/0:0/17:0), d5-DG(19:0/0:0/19:0), d5-TG(20:0/20:1/20:0), d5-TG(20:2/18:3/20:2), d5-TG(20:4/18:2/20:4), d5-DG(20:5/0:0/20:5), d5-TG(14:0/16:1/14:0), d5-TG(20:5/22:6/20:5), d5-TG(15:0/18:1/15:0),
d5-TG(16:0/18:0/16:0), d5-TG(17:0/17:1/17:0), d5-TG(19:0/12:0/19:0), d5-TG(20:0/20:1/20:0), d5-TG(20:2/18:3/20:2), d5-TG(20:4/18:2/20:4), $\operatorname{PE}(17: 0 / 14: 1)$, and $\operatorname{PC}(17: 0 / 14: 1)$.

2.4. Bioinformatics and Statistical Analyses. Nontargeted lipidomic analyses were performed as previously described, with some modifications [6]. For the UPLC-MS data, after normalizing against the dry weights, the resolved data sets were subjected to statistical analysis. The algorithm for significance analysis of microarray (SAM) data (the false discovery rate FDR $\leq 0.05$ unless otherwise noted) and unsupervised hierarchical clustering was performed using the web-based metabolomic data processing tool metaboAnalyst [40]. PCA and OPLS-DA were performed using SIMCA-P11.5. Other statistical calculations were conducted using PASW Statistics 20 (SPSS, Chicago, USA), and a $p$ value of 0.05 or less was considered significant in every comparison.

MUFA-to-PUFA ratios were calculated as previously described [26]. Briefly, adding levels of all MUFA lipids (lipids with one double bond in any acyl chain), resulting value was divided by the sum of all PUFA lipids (species with three or more double bonds in their acyl chains).

\section{Results}

3.1. Lipidomic Profile Associated with Aging in C. elegans. The first aim of the present study was to characterize changes in the lipidomic profile during aging in C. elegans to identify novel lipids or groups of lipids as biomarkers of aging. To this end, we acquired the nontargeted UPLC-HRMS lipidomic profiles of wild-type N2 in young adults (YA) (egg laying has not commenced) and day 10 adults (10A) (aging, egg laying has ceased). Selecting these two time points diminished interference from the egg laying and stochastic components of aging in C. elegans. Using these samples (approximately 3000 molecules), multivariate statistical analyses were performed, including unsupervised PCA [41] and supervised OPLS-DA [42] models. PCA and OPLS-DA score plots showed precise separation when the two groups were compared (Figures 1 and 2(b)). In addition, detailed lipid analysis suggested that in old worms the concentrations of DG, PC, and SM significantly decreased, while that of Cer increased (Figure S1). These results suggested that aging included the reprogramming of lipid metabolism.

3.2. Identification of a Lipid Set with respect to Age. We displayed the top 40 different lipids for $10 \mathrm{~A}$ worms compared with YA worms using heat mapping. The resulting heat map displayed a clear variation in the concentrations of metabolites with respect to age. Subsequently, to assess the individual discriminant of each component of the signature, $t$-test (2-tailed) or Mann-Whitney $U$ tests basing on checking for normal distribution were performed (data not shown).

Compared with YA worms, the concentration of longchain glycerol lipids (such as TG 57:3, TG 59:1, TG 58:3, and DG 42:3) increased in 10A worms, while the concentration of short-chain glycerol lipids (such as DG 30:0, TG 51:4, and DG 28:0) decreased. Additionally, glycerophospholipid 


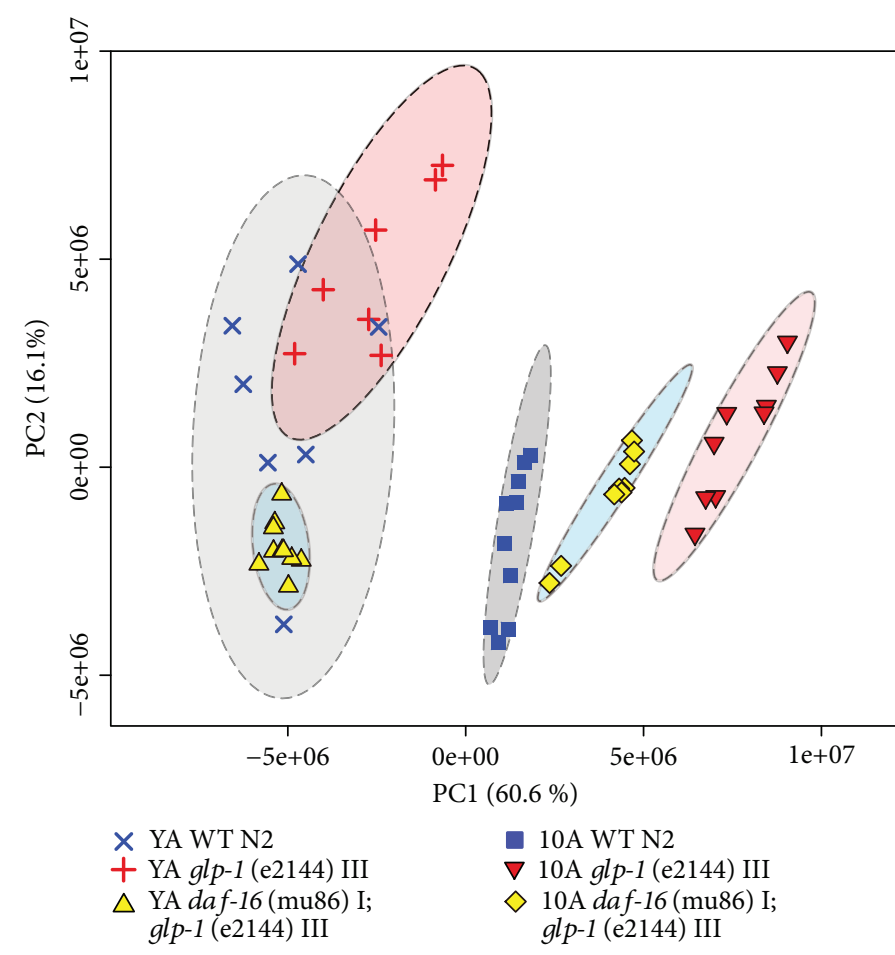

FIGURE 1: Lipid metabolic variations in WT, $g l p-1(e 2142)$, and daf16(mu86);glp-1(e2141) worms. PCA included young adults and 10 -day adults WT, $g l p-1$, and daf-16;glp-1. PC1 and PC2 represent the first and second principal components, respectively.

levels changed for selected species with advanced age, and the concentration of most PCs was decreased in aged worms (such as PC 29:5, PC 34:1, PC 29:1, and PC 28:2), and the concentrations of SM 32:0 and SM 34:2 were also decreased in aged animals (Figure 2(a)). In addition, consistent with a previous study on the lipidomics of familial longevity, these results showed that the concentrations of TG 57:2 and TG 56:7 significantly increased in aged animals (Figures 2(c) and 2(d)), although TG 56:6 did not (Figure 2(e)) [26].

Content differences in polyunsaturated (PUFA) and monounsaturated (MUFA) lipids determine membrane peroxidation, and the MUFA-to-PUFA ratio has been suggested to be a marker of longevity $[43,44]$. Therefore, we determined differences in the MUFA-to-PUFA ratio between YA and $10 \mathrm{~A}$ worms. 10A worms displayed a lower MUFA-toPUFA ratio compared with young worms (Figure 2(f)).

3.3. Lipidomic Characterization of Long-Lived glp-1 Mutants according to Longevity. Germ line elimination, leading to lifespan extension in C. elegans, is successfully simulated by mutations that cause GSC loss and sterility. One such temperature-sensitive mutant, glp-1(e2144ts), has been widely used as a model for lifespan extension resulting from germ line elimination. Fat metabolism, reproduction, and aging are intertwined regulatory axes, and previous studies have demonstrated that the longevity phenotype of the $g l p$ 1 mutant is associated with the regulation of lipid metabolism and lipid homeostasis using fat staining and GC to detect the fat content $[10,16,18]$. However, the specific lipids regulated by $g l p-1$ to extend lifespan remain unknown. To demonstrate the relationship between lipid metabolism and aging in the $g l p-1$ mutant, we detected the intact lipid profiles in the $g l p$ 1 mutant using a comprehensive lipidomic analysis method. Unsupervised PCA analysis showed that the $g l p-1$ mutant and wild-type samples have distinct lipid metabolic profiles at either the YA or 10A stage, albeit with little overlap at the young adult stage, which also indicated that aging increases metabolic differences (Figure 1). Further supervised OPLS-DA analysis revealed dramatic changes in the lipid metabolism of the $g l p-1$ mutant compared with the wild type at either the YA or 10A stage (Figures 3(a) and 3(b)).

Furthermore, the top 40 significantly different lipids of the YA and 10A $g l p-1$ mutants compared with wild-type worms were identified using heat mapping, and the results are listed in Figures 3(c) and 3(d). In addition, fat alterations associated with longevity primarily reflected the accumulation of short-chain lipids (such as TG 40:2, TG 40:1, and TG 41:1), which decreased in aged wild-type worms compared with young worms (Figures 3(c) and 3(d)). These finding suggested that the $g l p-1$ mutant regulated lipid metabolism to younger profiles compared with wild type.

Additionally, we used $\mathrm{RF}^{\mathrm{TM}}$ and univariate analyses to identify biomarkers associated with the long-lived phenotype and observed that $g l p-1$ dysregulated some age-variation lipids (such as PC 44:2, TG 48:4, and TG 50:1) to extend lifespan (Figure $4(\mathrm{c})$ ), although some lipids were not changed (such as DG 34:1, DG 42:3, PC 33:4, and TG 60:3). These results indicated that not all aspects of aging were reset in long-living $g l p-1$ mutants. More detailed information regarding the significant metabolite changes in $g l p-1$ mutants compared with wild-type worms are listed in the supplemental information, Tables S1 and S2. In addition, the agevariation MUFA-to-PUFA ratio was obviously increased in $g l p-1$ mutants compared with wild type at either the YA or 10A stage (Figures 5(b) and 5(c)).

3.4. FOXO/DAF-16 Mediate Lipid Metabolism Reprogramming in glp-1 Mutants. Previous studies have demonstrated that the transcription regulators DAF16/FOXO are essential for the longevity of germ line-less adults, enhancing the expression of multiple genes involved in lipid metabolism and gene classes that promote longevity $[16,18]$. Here, we used lipidomic analysis to reveal how germ line loss mutants depend on DAF-16 to regulate lipid metabolism to achieve the longevity phenotype. The results of multivariate analysis, including PCA and OPLS-DA, revealed that the lipid metabolism profiles of daf-16;glp-1 mutants were different from those of wild type and the $g l p-1$ mutant (data not shown). Further univariate analysis indicated that some age-related metabolites regulated by germ line loss signals were daf-16-dependent (such as DG 34:3; TG 49:2, and TG 51:3) (Figure 5(a)), while other metabolites showed more complex epistatic patterns, such as TG 46:5, TG 40:1, and PC $38: 3$. More detailed information is provided in the supplemental information, Tables S1 and S2. In addition, the agerelated MUFA-to-PUFA ratio was upregulated in the $g l p-1$ mutant, while no variation was observed in the daf-16;glp-1 mutant, which may indicate that the age-related MUFA-to- 


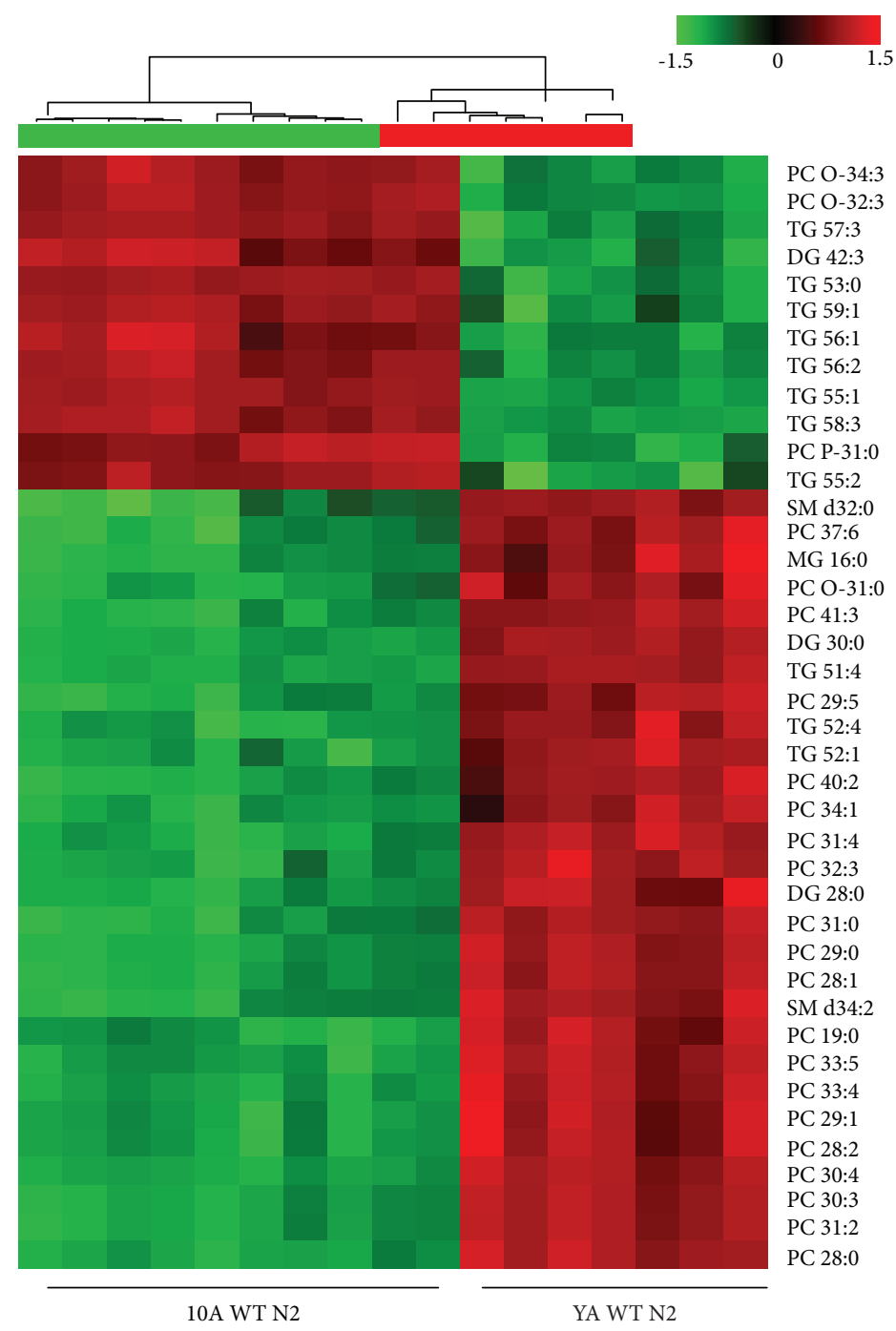

(a)
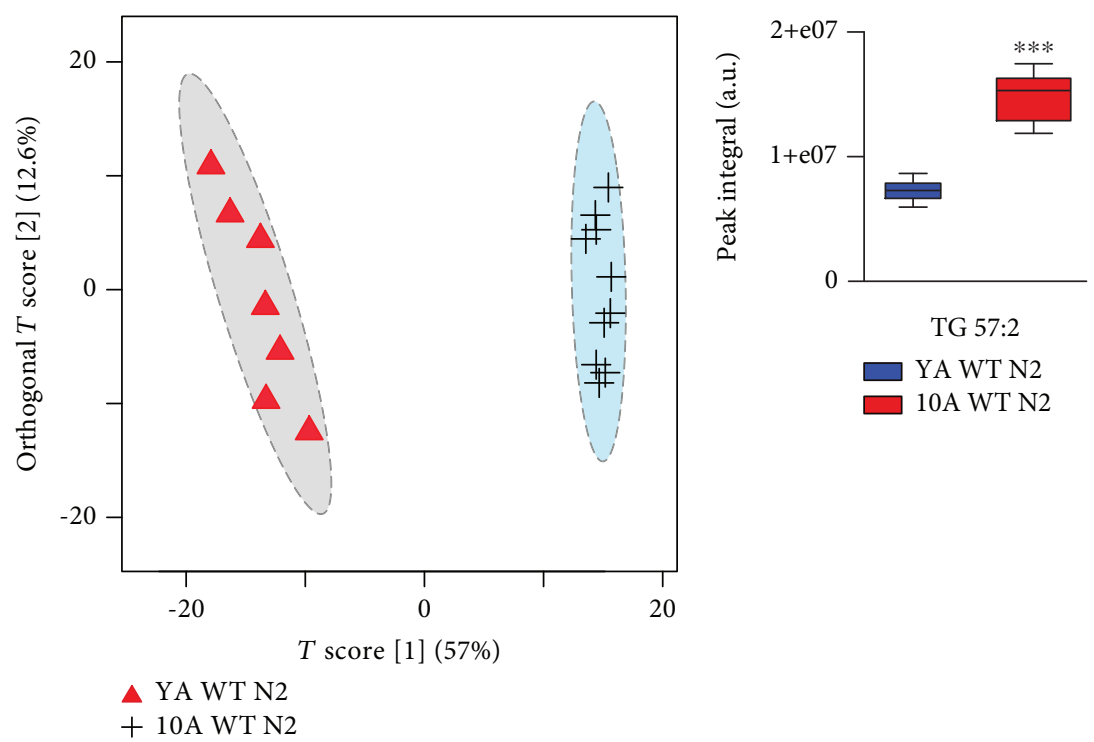

(b)

(c)

FIGURe 2: Continued. 


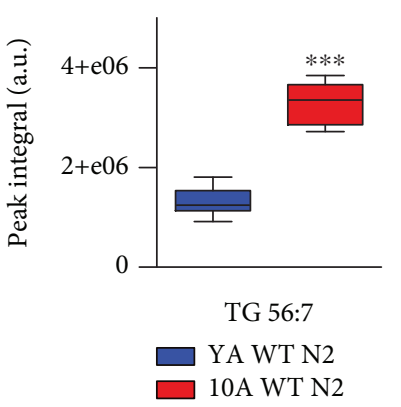

(d)

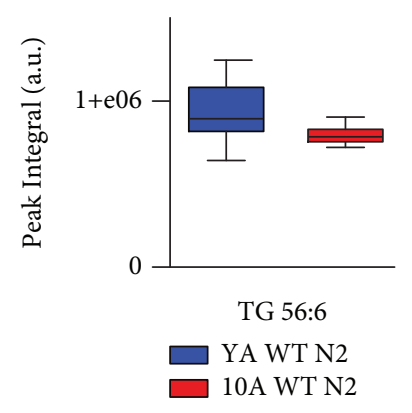

(e)

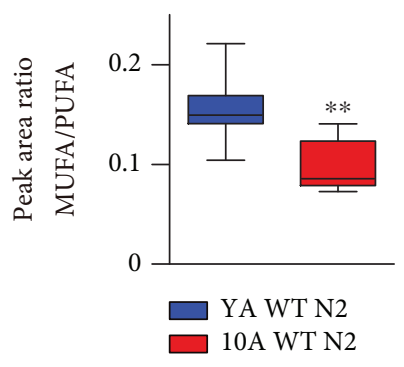

(f)

FIGURE 2: Age-related comprehensive lipidomic analysis in wild-type C. elegans (a) lipidomic analysis for YA (young adult) and 10A (10 days of adulthood) wild-type N2. Heat map plot showing the 40 most important different metabolites from the samples according to their aging status. Data are presented using hierarchical clustering (Pearson's correlation coefficient). Metabolite abundance levels were reflected in the heat maps using colors, with blue representing lower and red representing higher levels when comparing the mean metabolite abundance values. The distance function 1-correlation was used in hierarchical clustering to determine the order of metabolites and animals. (b) Scores from OPLS-DA model for YA and 10A wild-type N2. The peak integral (a.u.) of (c) TG 57:2, (d) TG 56:7, and (e) TG 56:6 in YA $\mathrm{N} 2$ and $10 \mathrm{~A}$ N2. (f) MUFA-to-PUFA ratio differences in YA N2 and 10A N2. The $p$ values were calculated using the Mann-Whitney $U$ test, and 0.05 or less was considered significant. All statistical analyses were conducted using SPSS package.

PUFA ratio regulation in $g l p-1$ was mediated by daf-16 (Figures 5(b), 5(c)-5(e)).

\section{Discussion}

Untargeted lipidomics enables the comprehensive investigation of endogenous lipids in complex biological systems and has the potential to identify modifications in metabolic pathways and networks in response to biological effects [45, 46]. The role of lipids in aging diseases and human longevity has been widely acknowledged, and the metabolic changes of several lipids associated with age-related diseases, such as hypertension, diabetes type 2, and cardiovascular disease, have been identified [47-49]. Therefore, we investigated the aging-related lipid metabolic variation in C. elegans. Analysis of the age-variation lipid profiles in aged worms revealed that the levels of DG, PC, and SM were significantly decreased, while that of Cer was increased. In addition, these results showed that accumulation of long-chain lipid and deleterious short-chain lipids in aged worms might contribute to aging. The aging-related lipid profile was also characterized by a lower MUFA-to-PUFA ratio. The present study is the first to report the age-related lipidomic profiles in C. elegans. The lipid profile, representative of healthy aging in C. elegans, comprised higher levels of ether PC and SM species and lower levels of PE and long-chain TG species.

In the present study, we observed that the levels of some SM significantly decreased, while those of Cer increased in aged worms compared with young animals. Sphingomyelin species are important components of tissue membranes and cellular messengers and are particularly abundant in neuronal cells. Low levels of SM species have been associated with aging-related diseases, including $\mathrm{Alz}$ heimer's, Parkinson's, Huntington's [50], diabetes [30], subclinical atherosclerosis [51], and cardiovascular disease [52]. Sphingomyelinase (SMase) is an enzyme that hydrolyzes SM species into ceramides, whose activity tends to increase with age, thereby decreasing SM levels and increasing ceramide levels $[53,54]$. In addition, consistent with the results of the present study, a recent lipidomic study of familial longevity and aging kidney uncovered that the levels of SM species decreased in aged individuals $[26,27]$. Thus, alterations in ceramide metabolism may directly influence aging.

We also detected decreasing levels of PC species. PC species prevent the oxidation of polyunsaturated fatty acids in lipoproteins [55]. In addition, upregulation of ether phospholipids is associated with a lower risk for hypertension, diabetes, and aging kidney [27, 30]. In addition, consistent with the results of the present study, a familial longevity study revealed that a higher level of $\mathrm{PC}$ represents a younger lipid profile [26]. Thus, PC species were present at lower levels in aged worms, consistent with the decreased antioxidant capacity of these molecules.

Long-chain triglycerides undergo beta-oxidation or peroxisomes, whose enzymatic capacity decreases with age 


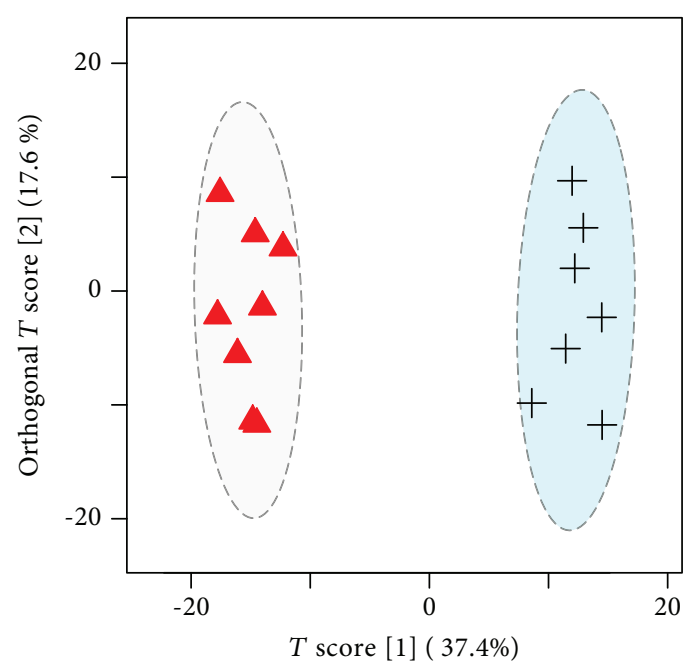

A YA glp-1

+ YA N2

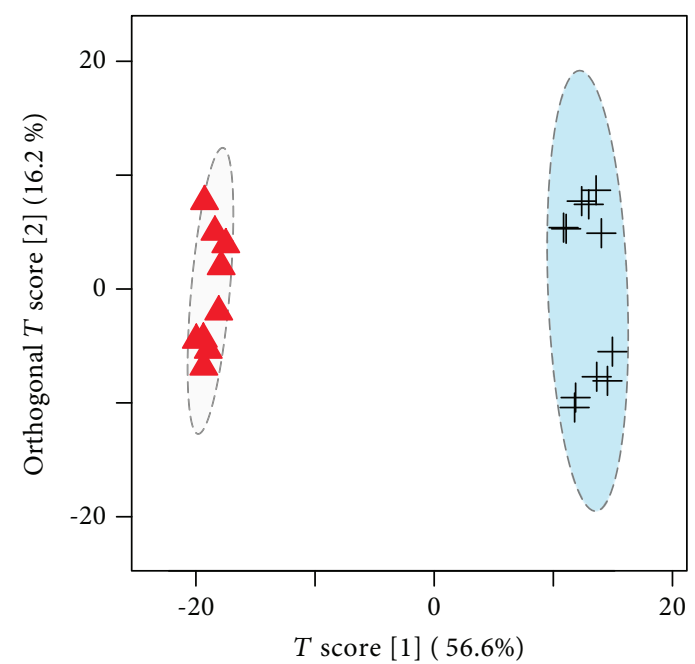

A 10A glp-1

$+10 \mathrm{~A} \mathrm{~N} 2$

(a)

(b)
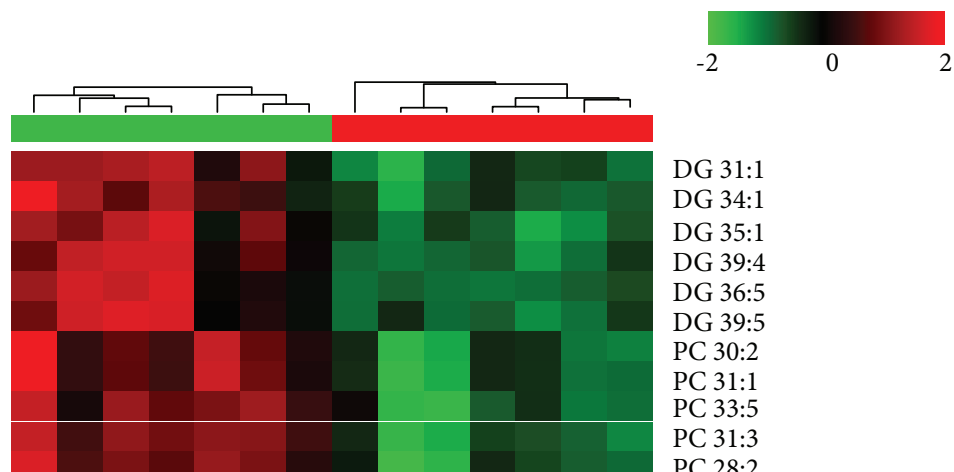

DG 31:1

DG 34:1

DG 35:1

DG 39:4

DG 36:5

DG 39:5

PC 30:2

PC 31:1

PC 33:5

PC $31: 3$

PC 28:2

PC 33:4

PC 29:3

TG $48: 2$

TG 47:0

TG 46:0

TG 44:0

TG 48:1

TG 48:2

DG 42:9

PC 40:0

TG 53:4

TG 47:1

TG 51:1

TG 49:1

PC 42:0

TG 57:7

DG 42:3

TG 41:1

TG 40:2

TG 40:0

TG 42:0

Cer d46:0

TG 41:0

TG 43:0

TG 59:2

TG 62:5

TG 62:3

TG 59:1

SM d 40:3

YA WT N2

YA glp-1(e2144) III

(c)

Figure 3: Continued. 

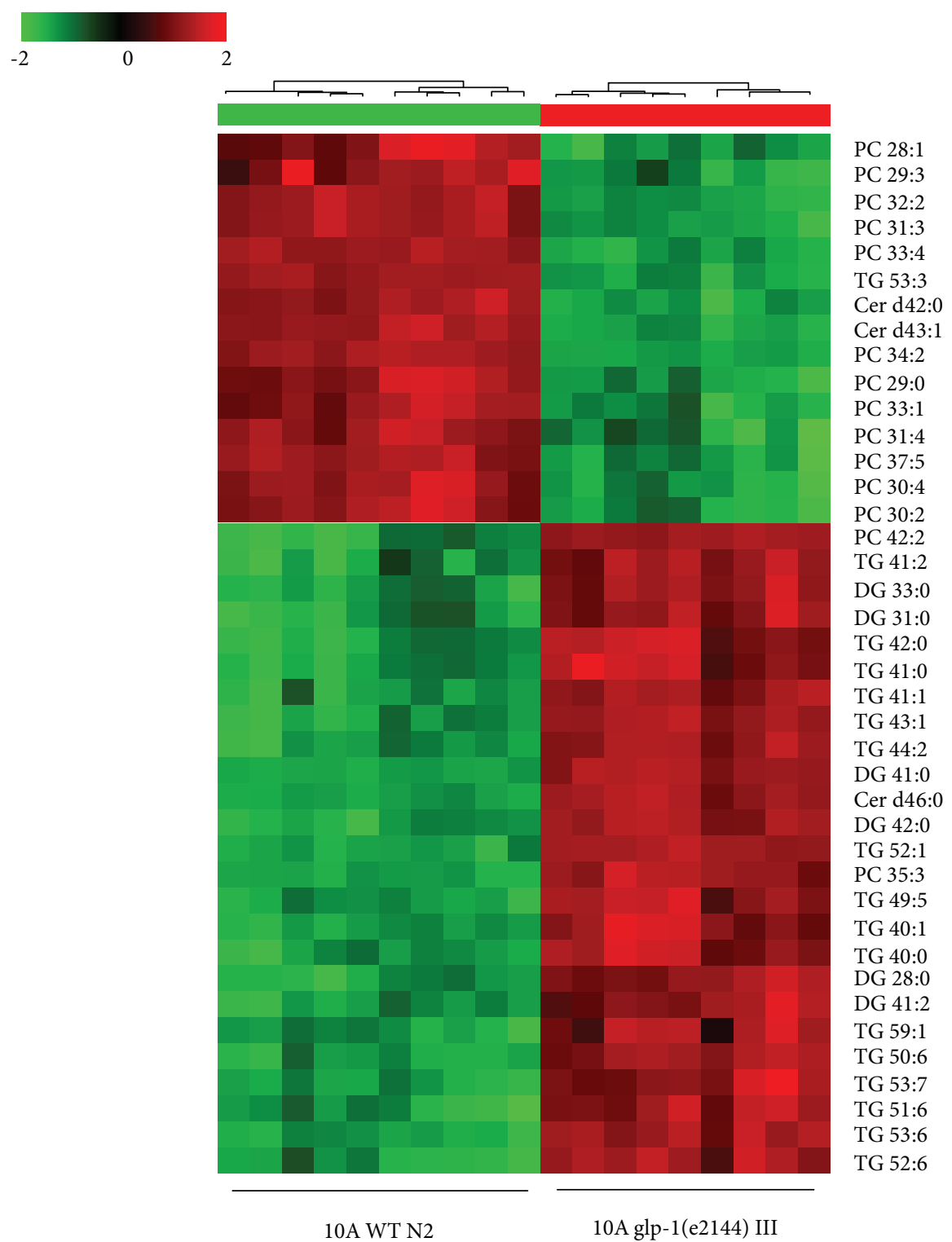

(d)

FIGURE 3: The long-lived $g l p-1(e 2144)$ mutant has a distinctive lipid metabolic profile. (a) Scores from the OPLS-DA model for $g l p-1$ and wildtype N2 at (a) young adult stage and (b) 10 days of adulthood. Heat map plot showed the top 40 most important different metabolites from glp-1 against $\mathrm{N} 2$ at (c) young adult stage and (d) 10 days of adulthood.

[26]. Therefore, in aged worms, higher levels of long-chain TG and lower level of short-chain TG may reflect a declined beta-oxidation function compared to younger worms.

The fact that the MUFA-to-PUFA ratio is a longevity biomarker is consistent with reports showing a negative correlation between the membrane double bond content and longevity in mammals $[56,57]$. SFA and MUFA are more essentially resistant to peroxidation than PUFA; thus, higher PUFA levels may increase lipid peroxidation and oxidative damage $[56,58]$. Thus, in aged worms, a lower MUFA-to-PUFA ratio may be more prone to lipid peroxidation and oxidative damage. Altogether, the lipidome of aged worms, with a lower PC and higher polyunsaturated TG species, may reflect the accumulation of oxidative damage in aged animals.
A previous study reported that $g l p-1$ mutants showed increased fat storage at the nonpermissive temperature relative to the wild type (N2) by using oil red O staining [16]. Other transcriptomic studies revealed that many aspects of lipid metabolism were altered in $g l p-1$ mutants, including fatty acid (FA) oxidation, FA desaturation, and triglyceride lipase $[16,18]$. Another study showed that some FA (i.e., C16:0, C16:1n7, C18:0, and C18:3) changed in glp-1 mutant through GC analysis $[16,18]$. However, these studies did not determine which intact lipids were regulated by $g l p-1$ to achieve lifespan extension (Figures 4(a) and 4(b)), although total fatty acid analysis can provide insight into fatty acid metabolism. Extending upon previous studies, we analyzed the lipidome of $g l p-1$ mutants. Consistent with the results of a previous study, these results demonstrated that fat 


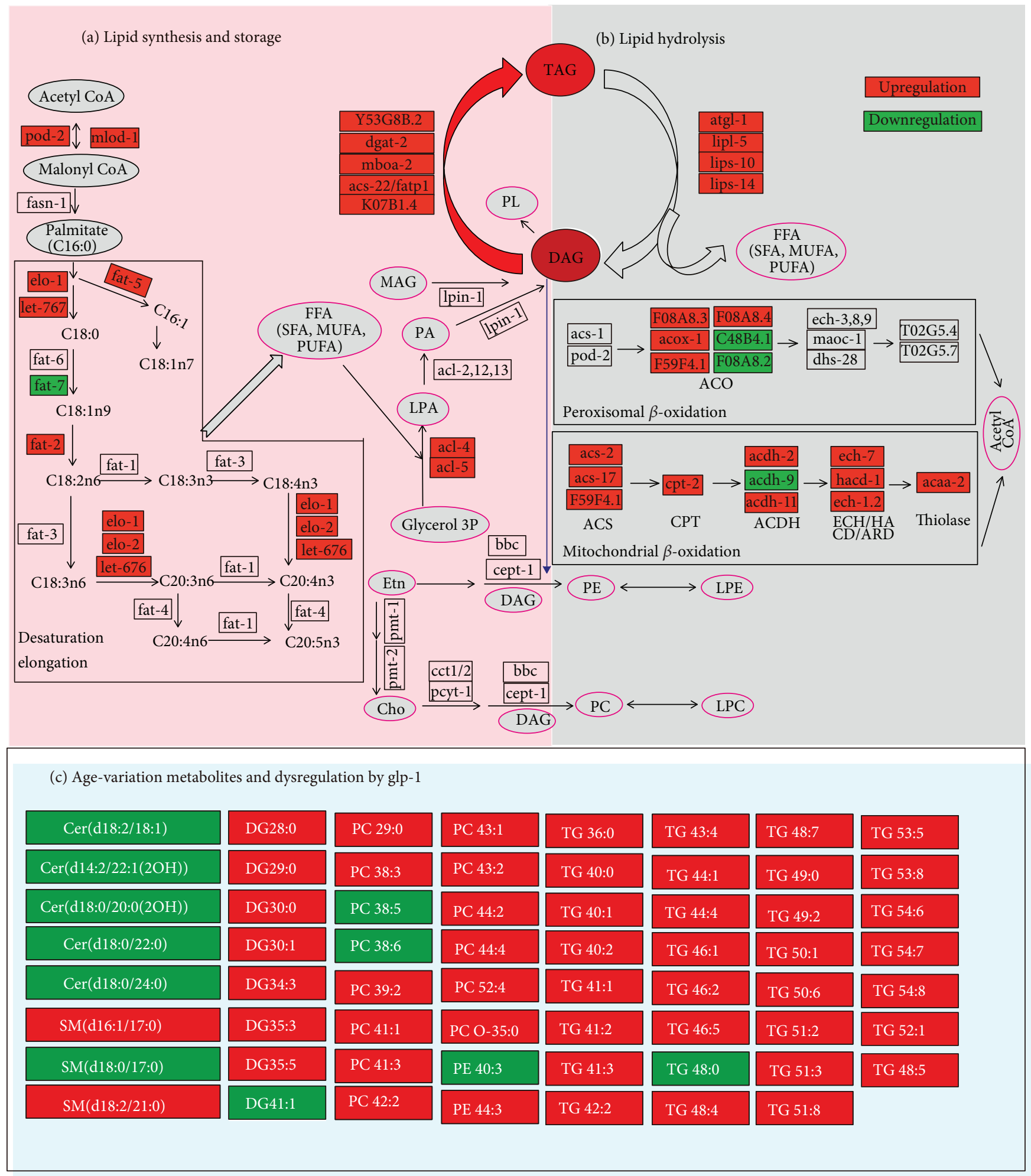

FIGURE 4: $g l p-1$ regulated age-variation lipid to achieve the longevity phenotype. (a, b) $g l p-1$ regulated the key genes involved in lipid anabolism and catabolism, which was modified from Amrit et al., PLoS Genetics 2016 [16]. (c) glp-1 regulated age-variation lipid, and the colors reflect lipid changes, with yellow representing lower and red representing higher values when comparing $g l p-1$ mutants vs. N2.

storage elevated in $g l p-1$ mutants. Compared to previous studies, we observed more special intact lipid molecule information regulated by $g l p-1$. We observed that $g l p-1$ regulated some age-related lipids to extend lifespan, including PC 44:2, TG 48:4, and TG50:1 (Figures 4(c) and 5(a)). Furthermore, analysis of the lipidomics of the daf-16;glp-1 double 


\begin{tabular}{|c|c|c|c|c|c|}
\hline Matabolite & $\begin{array}{l}\text { WT: } \\
\text { 10AVS YA }\end{array}$ & $\begin{array}{l}\text { YA: } \\
\text { glp-1 VS WT }\end{array}$ & $\begin{array}{l}\text { 10A: } \\
\text { glp-1 VS WT }\end{array}$ & $\begin{array}{l}\text { YA: } \\
\text { glp-1;daf-16 } \\
\text { VS WT }\end{array}$ & $\begin{array}{l}\text { 10A: } \\
\text { glp-1;daf-16 } \\
\text { VS WT }\end{array}$ \\
\hline \multicolumn{6}{|l|}{ CerP d26:1 } \\
\hline \multicolumn{6}{|l|}{ DG 28:0 } \\
\hline \multicolumn{6}{|l|}{ DG 29:0 } \\
\hline \multicolumn{6}{|l|}{ DG 30:0 } \\
\hline \multicolumn{6}{|l|}{ DG 30:1 } \\
\hline \multicolumn{6}{|l|}{ DG 34:3 } \\
\hline \multicolumn{6}{|l|}{ DG 36:5 } \\
\hline \multicolumn{6}{|l|}{ PC 52:4 } \\
\hline \multicolumn{6}{|l|}{ PC 31:2 } \\
\hline \multicolumn{6}{|l|}{ PC 39:2 } \\
\hline \multicolumn{6}{|l|}{ PC 40:4 } \\
\hline \multicolumn{6}{|l|}{ PC 44:2 } \\
\hline \multicolumn{6}{|l|}{ TG 48:4 } \\
\hline \multicolumn{6}{|l|}{ TG 48:5 } \\
\hline \multicolumn{6}{|l|}{ TG 49:2 } \\
\hline \multicolumn{6}{|l|}{ TG 50:1 } \\
\hline \multicolumn{6}{|l|}{ TG 50:6 } \\
\hline \multicolumn{6}{|l|}{ TG 51:2 } \\
\hline \multicolumn{6}{|l|}{ TG 51:3 } \\
\hline \multicolumn{6}{|l|}{ TG 51:4 } \\
\hline \multicolumn{6}{|l|}{ TG 51:8 } \\
\hline \multicolumn{6}{|l|}{ TG 52:0 } \\
\hline \multicolumn{6}{|l|}{ TG 52:1 } \\
\hline \multicolumn{6}{|l|}{ TG 52:2 } \\
\hline \multicolumn{6}{|l|}{ TG 53:5 } \\
\hline \multicolumn{6}{|l|}{ TG 53:9 } \\
\hline \multicolumn{6}{|l|}{ TG 56:0 } \\
\hline \multicolumn{6}{|l|}{ TG 57:10 } \\
\hline \multicolumn{6}{|l|}{ TG 57:9 } \\
\hline \multicolumn{6}{|l|}{ TG 58:0 } \\
\hline TG 61:4 & & & & & \\
\hline TG 62:4 & & & & & \\
\hline
\end{tabular}

(a)

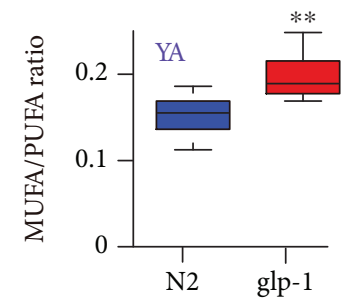

(b)

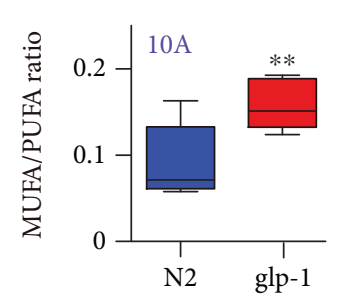

(c)

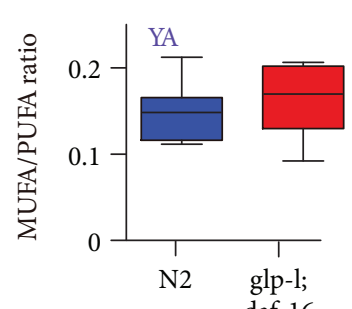

(d)

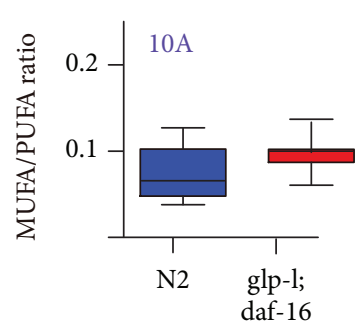

(e)

FIgURE 5: The age-related metabolic variation regulated by $g l p-1$ partly depends on FOXO/DAF-16. (a) Metabolite variation with age in $g l p-$ 1(e2144) and daf-16(mu86);glp-1(e2144) double mutants in young adults and 10-day-old adults. Metabolite abundance levels were reflected using colors, increase (green) or decrease (purple) in metabolite concentrations, gray represents no significant difference, when mutants vs. N2. MUFA-to-PUFA ratio differences when $g l p-1$ vs. N2 at (b) young adult stage or (c) 10 -day-old adult stage, or when daf-16;glp-1 vs. N2 at (d) young adult stage or (e) 10-day-old adult. $p$ values that were calculated using the Mann-Whitney $U$ test of 0.05 or less was considered significant. All statistical analyses were calculated using SPSS package. $p$ values were calculated using the Mann-Whitney $U$ test, and the $p$ value of 0.05 or less was considered significant. All statistical analyses were calculated using SPSS package. 


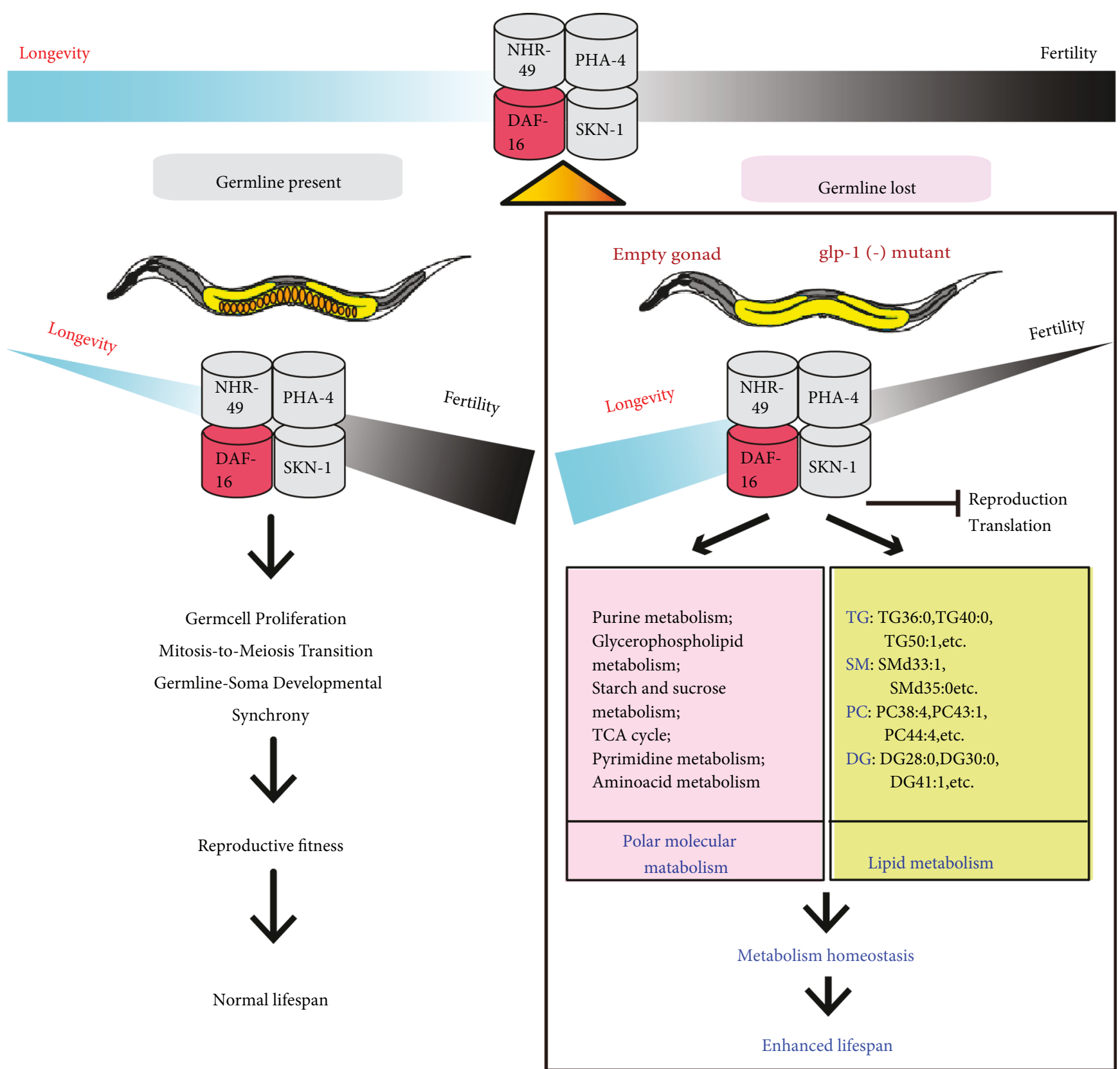

FIGURE 6: Metabolomic and lipidomic signatures with reproduction-regulated aging in Caenorhabditis elegans.

mutant revealed that DAF-16 partially mediated $g l p-1$-regulated age-variation lipid metabolism. Indeed, a previous study reported that other downstream target genes also mediated $g l p-1$ to regulate lipid metabolism to extend lifespan, such as SKN-1 [18], TCER-1 [16], and NHR-49 [17]. Combined with the results of a previous metabolomic studies, there was an inseparable relationship between the extension of the lifespan of $g l p-1$ and aging-related metabolic changes (Figure 6).

To our knowledge, the present study is the first to use a comprehensive high-resolution lipidome to reveal the relationship between longevity and lipid metabolism. While there are some drawbacks of the lipidomic method, we obtained putative matches for lipids based on databases and references, but these matches often carry considerable uncertainty. In some cases, a single mass-charge ratio may match more than 10 putative known molecules. In addition, some lipids (such as TG (18:1/18:1/18:0) and TGs (18:0/18:2/18:0)) share the same molecular formulas and molecular weights. Thus, the only difference between these molecules is the position of the double bond. These molecules are typically considered to be TGs (54:2). Thus, it is difficult to obtain accurate lipid structure information from lipidomic studies. Moreover, it is difficult to conduct lipid metabolic set enrichment analysis, reflecting the relatively few known lipid metabolic pathways. Therefore, 
the present study likely represents only a variation of individual lipid molecules during aging but not the lipid metabolic pathway.

Nevertheless, the results of the present study show the power of using lipidomics to better understand the phenotype of aging and longevity in C. elegans. In future studies of aging mechanisms, this knowledge could be linked to functional studies. In any case, the findings of the present study, consistent with the available evidence, clearly suggest that lipid metabolism is closely associated with the aging process and that the long-lived phenotype of $g l p-1$ is closely related to the regulation of lipid metabolism.

\section{Data Availability}

All the figures and tables used to support the findings of this study are included within the article and supplementary materials.

\section{Conflicts of Interest}

The authors declare that there is no conflict of interest.

\section{Acknowledgments}

We would like to thank the Caenorhabditis Genetics Center (CGC) for providing the worm strains, which is funded by NIH Office of Research Infrastructure Programs (P40OD010440). Financial support was received from the Natural Science Foundation of China (81771516, 81801398, and 81671405) and the Science and Technology Cooperation Project of Luzhou and Southwest Medical University (2016LZXNYD-Z05).

\section{Supplementary Materials}

Figure S1: heat map plot listed the lipid variation between YA and 10A N2. Table S1: list of altered lipids of each mutant strain against the wild type in young adults. Table S2: list of altered lipids of each mutant strain against the wild-type days 10 of adult worms. (Supplementary Materials)

\section{References}

[1] L. Ferrucci, F. Giallauria, and J. M. Guralnik, "Epidemiology of aging," Radiologic Clinics of North America, vol. 46, no. 4, pp. 643-652, 2008.

[2] R. N. Butler, R. A. Miller, D. Perry et al., "New model of health promotion and disease prevention for the 21st century," BMJ, vol. 337, article a399, 2008.

[3] C. A. Wijsman, M. P. Rozing, T. C. M. Streefland et al., "Familial longevity is marked by enhanced insulin sensitivity," Aging Cell, vol. 10, no. 1, pp. 114-121, 2011.

[4] C. López-Otín, L. Galluzzi, J. M. P. Freije, F. Madeo, and G. Kroemer, "Metabolic control of longevity," Cell, vol. 166, no. 4, pp. 802-821, 2016.

[5] R. Pamplona and G. Barja, "An evolutionary comparative scan for longevity-related oxidative stress resistance mechanisms in homeotherms," Biogerontology, vol. 12, no. 5, pp. 409-435, 2011.
[6] Q.-L. Wan, X. Shi, J. Liu et al., "Metabolomic signature associated with reproduction-regulated aging in Caenorhabditis elegans," Aging, vol. 9, no. 2, pp. 447-474, 2017.

[7] H. Hsin and C. Kenyon, "Signals from the reproductive system regulate the lifespan of C. elegans," Nature, vol. 399, no. 6734, pp. 362-366, 1999.

[8] N. Arantes-Oliveira, J. Apfeld, A. Dillin, and C. Kenyon, "Regulation of life-span by germ-line stem cells in Caenorhabditis elegans," Science, vol. 295, no. 5554, pp. 502-505, 2002.

[9] L. R. Lapierre, S. Gelino, A. Meléndez, and M. Hansen, "Autophagy and lipid metabolism coordinately modulate life span in germline-less C. elegans," Current Biology, vol. 21, no. 18, pp. 1507-1514, 2011.

[10] M. C. Wang, E. J. O'rourke, and G. Ruvkun, "Fat metabolism links germline stem cells and longevity in C. elegans," Science, vol. 322, no. 5903, pp. 957-960, 2008.

[11] E. T. Judd, F. J. Wessels, M. D. Drewry et al., "Ovariectomy in grasshoppers increases somatic storage, but proportional allocation of ingested nutrients to somatic tissues is unchanged," Aging Cell, vol. 10, no. 6, pp. 972-979, 2011.

[12] E. Thomsen and K. Hamburger, "Oxygen consumption of castrated females of the blow-fly, Calliphora erythrocephala Meig," Journal of Experimental Biology, vol. 32, no. 4, pp. 692-699, 1955.

[13] E. Pallier, R. Aubert, and D. Lemonnier, "Effect of diet and ovariectomy on adipose tissue cellularity in mice," Reproduction Nutrition Développement, vol. 20, no. 3A, pp. 631-636, 1980.

[14] M. J. Fettman, C. A. Stanton, L. L. Banks et al., "Effects of neutering on bodyweight, metabolic rate and glucose tolerance of domestic cats," Research in Veterinary Science, vol. 62, no. 2, pp. 131-136, 1997.

[15] S. W. Crane, "Occurrence and management of obesity in companion animals," Journal of Small Animal Practice, vol. 32, no. 6, pp. 275-282, 1991.

[16] F. R. G. Amrit, E. M. Steenkiste, R. Ratnappan et al., "DAF-16 and TCER-1 facilitate adaptation to germline loss by restoring lipid homeostasis and repressing reproductive physiology in C. elegans," PLoS Genetics, vol. 12, no. 2, article e1005788, 2016.

[17] R. Ratnappan, F. R. G. Amrit, S. W. Chen et al., "Germline signals deploy NHR-49 to modulate fatty-acid $\beta$-oxidation and desaturation in somatic tissues of C. elegans," PLoS Genetics, vol. 10, no. 12, article e1004829, 2014.

[18] M. J. Steinbaugh, S. D. Narasimhan, S. Robida-Stubbs et al., "Lipid-mediated regulation of SKN-1/Nrf in response to germ cell absence," eLife, vol. 4, article e07836, 2015.

[19] M. McCormick, K. Chen, P. Ramaswamy, and C. Kenyon, "New genes that extend Caenorhabditis elegans' lifespan in response to reproductive signals," Aging Cell, vol. 11, no. 2, pp. 192-202, 2012.

[20] X. Han, K. Yang, and R. W. Gross, "Multi-dimensional mass spectrometry-based shotgun lipidomics and novel strategies for lipidomic analyses," Mass Spectrometry Reviews, vol. 31, no. 1, pp. 134-178, 2012.

[21] C. S. Ejsing, J. L. Sampaio, V. Surendranath et al., "Global analysis of the yeast lipidome by quantitative shotgun mass spectrometry," Proceedings of the National Academy of Sciences, vol. 106, no. 7, pp. 2136-2141, 2009.

[22] E. Szymańska, F. A. van Dorsten, J. Troost et al., “A lipidomic analysis approach to evaluate the response to cholesterollowering food intake," Metabolomics, vol. 8, no. 5, pp. 894906, 2012. 
[23] R. B. Chan, T. G. Oliveira, E. P. Cortes et al., "Comparative lipidomic analysis of mouse and human brain with Alzheimer disease," Journal of Biological Chemistry, vol. 287, no. 4, pp. 2678-2688, 2012.

[24] C. Hu, H. Wei, A. M. van den Hoek et al., "Plasma and liver lipidomics response to an intervention of rimonabant in ApoE* 3Leiden. CETP transgenic mice," PLoS One, vol. 6, no. 5, p. e19423, 2011.

[25] H. K. Min, S. Lim, B. C. Chung, and M. H. Moon, "Shotgun lipidomics for candidate biomarkers of urinary phospholipids in prostate cancer," Analytical and Bioanalytical Chemistry, vol. 399, no. 2, pp. 823-830, 2011.

[26] V. Gonzalez-Covarrubias, M. Beekman, H. W. Uh et al., "Lipidomics of familial longevity," Aging Cell, vol. 12, no. 3, pp. 426-434, 2013.

[27] F. Braun, M. M. Rinschen, V. Bartels et al., "Altered lipid metabolism in the aging kidney identified by three layered omic analysis," Aging (Albany NY), vol. 8, no. 3, pp. 441-454, 2016.

[28] I. Montoliu, M. Scherer, F. Beguelin et al., "Serum profiling of healthy aging identifies phospho-and sphingolipid species as markers of human longevity," Aging (Albany NY), vol. 6, no. 1, pp. 9-25, 2014.

[29] J. Graessler, D. Schwudke, P. E. H. Schwarz, R. Herzog, A. Shevchenko, and S. R. Bornstein, "Top-down lipidomics reveals ether lipid deficiency in blood plasma of hypertensive patients," PLoS One, vol. 4, no. 7, article e6261, 2009.

[30] K. Suhre, C. Meisinger, A. Döring et al., "Metabolic footprint of diabetes: a multiplatform metabolomics study in an epidemiological setting," PLoS One, vol. 5, no. 11, article e13953, 2010.

[31] S. Brenner, "The genetics of Caenorhabditis elegans," Genetics, vol. 77, no. 1, pp. 71-94, 1974.

[32] S. K. Davies, A. M. Leroi, and J. G. Bundy, "Fluorodeoxyuridine affects the identification of metabolic responses to daf-2 status in Caenorhabditis elegans," Mechanisms of Ageing and Development, vol. 133, no. 1, pp. 46-49, 2012.

[33] M. Witting, T. V. Maier, S. Garvis, and P. Schmitt-Kopplin, "Optimizing a ultrahigh pressure liquid chromatographytime of flight-mass spectrometry approach using a novel sub$2 \mu \mathrm{m}$ core-shell particle for in depth lipidomic profiling of Caenorhabditis elegans," Journal of Chromatography A, vol. 1359, pp. 91-99, 2014.

[34] A. Seyer, S. Boudah, S. Broudin, C. Junot, and B. Colsch, "Annotation of the human cerebrospinal fluid lipidome using high resolution mass spectrometry and a dedicated data processing workflow," Metabolomics, vol. 12, no. 5, p. 91, 2016.

[35] E. Fahy, M. Sud, D. Cotter, and S. Subramaniam, "LIPID MAPS online tools for lipid research," Nucleic Acids Research, vol. 35, Web Server, pp. W606-W612, 2007.

[36] E. Yasugi and K. Watanabe, "LIPIDBANK for Web, the newly developed lipid database," Tanpakushitsu Kakusan Koso. Protein, Nucleic Acid, Enzyme, vol. 47, no. 7, pp. 837-841, 2002.

[37] D. S. Wishart, D. Tzur, C. Knox et al., "HMDB: the human metabolome database," Nucleic Acids Research, vol. 35, Database, pp. D521-D526, 2007.

[38] J. M. Foster, P. Moreno, A. Fabregat et al., "LipidHome: a database of theoretical lipids optimized for high throughput mass spectrometry lipidomics," PLoS One, vol. 8, no. 5, article e61951, 2013.

[39] M. Caffrey and J. Hogan, "LIPIDAT: a database of lipid phase transition temperatures and enthalpy changes. DMPC data subset analysis," Chemistry and Physics of Lipids, vol. 61, no. 1, pp. 1-109, 1992.

[40] J. Xia, I. V. Sinelnikov, B. Han, and D. S. Wishart, "MetaboAnalyst 3.0-making metabolomics more meaningful," Nucleic Acids Research, vol. 43, no. W1, pp. W251-W257, 2015.

[41] S. Wold, K. Esbensen, and P. Geladi, "Principal component analysis," Chemometrics and Intelligent Laboratory Systems, vol. 2, no. 1-3, pp. 37-52, 1987.

[42] J. Trygg and S. Wold, "Orthogonal projections to latent structures (O-PLS)," Journal of Chemometrics, vol. 16, no. 3, pp. 119-128, 2002.

[43] P. Caprari, A. Scuteri, A. M. Salvati et al., "Aging and red blood cell membrane: a study of centenarians," Experimental Gerontology, vol. 34, no. 1, pp. 47-57, 1999.

[44] R. Pamplona, M. Portero-Otín, D. Riba et al., "Mitochondrial membrane peroxidizability index is inversely related to maximum life span in mammals," Journal of Lipid Research, vol. 39, no. 10, pp. 1989-1994, 1998.

[45] J. K. Nicholson, J. C. Lindon, and E. Holmes, “Metabonomics': understanding the metabolic responses of living systems to pathophysiological stimuli via multivariate statistical analysis of biological NMR spectroscopic data," Xenobiotica, vol. 29, no. 11, pp. 1181-1189, 1999.

[46] O. Fiehn, "Metabolomics-the link between genotypes and phenotypes," Plant Molecular Biology, vol. 48, no. 1/2, pp. 155-171, 2002.

[47] J. Yeboah, C. McNamara, X. C. Jiang et al., "Association of plasma sphingomyelin levels and incident coronary heart disease events in an adult population," Arteriosclerosis, Thrombosis, and Vascular Biology, vol. 30, no. 3, pp. 628-633, 2010.

[48] A. C. I. Boullart, J. de Graaf, and A. F. Stalenhoef, "Serum triglycerides and risk of cardiovascular disease," Biochimica et Biophysica Acta (BBA) - Molecular and Cell Biology of Lipids, vol. 1821, no. 5, pp. 867-875, 2012.

[49] M. Miller, N. J. Stone, C. Ballantyne et al., "Triglycerides and cardiovascular disease," Circulation, vol. 123, no. 20, pp. 2292-2333, 2011.

[50] M. Piccinini, F. Scandroglio, S. Prioni et al., "Deregulated sphingolipid metabolism and membrane organization in neurodegenerative disorders," Molecular Neurobiology, vol. 41, no. 2-3, pp. 314-340, 2010.

[51] J. C. Nelson, X. C. Jiang, I. Tabas, A. Tall, and S. Shea, "Plasma sphingomyelin and subclinical atherosclerosis: findings from the multi-ethnic study of atherosclerosis," American Journal of Epidemiology, vol. 163, no. 10, pp. 903-912, 2006.

[52] W. L. Holland and S. A. Summers, "Sphingolipids, insulin resistance, and metabolic disease: new insights from in vivo manipulation of sphingolipid metabolism," Endocrine Reviews, vol. 29, no. 4, pp. 381-402, 2008.

[53] E. L. Smith and E. H. Schuchman, "The unexpected role of acid sphingomyelinase in cell death and the pathophysiology of common diseases," The FASEB Journal, vol. 22, no. 10, pp. 3419-3431, 2008.

[54] A. R. Smith, F. Visioli, B. Frei, and T. M. Hagen, "Age-related changes in endothelial nitric oxide synthase phosphorylation and nitric oxide dependent vasodilation: evidence for a novel mechanism involving sphingomyelinase and ceramideactivated phosphatase 2A," Aging Cell, vol. 5, no. 5, pp. 391400, 2006.

[55] P. Wiesner, K. Leidl, A. Boettcher, G. Schmitz, and G. Liebisch, "Lipid profiling of FPLC-separated lipoprotein fractions by 
electrospray ionization tandem mass spectrometry," Journal of Lipid Research, vol. 50, no. 3, pp. 574-585, 2009.

[56] R. Pamplona, M. Portero-Otín, C. Ruiz, R. Gredilla, A. Herrero, and G. Barja, "Double bond content of phospholipids and lipid peroxidation negatively correlate with maximum longevity in the heart of mammals," Mechanisms of Ageing and Development, vol. 112, no. , pp. 169-183, 2000.

[57] T. W. Mitchell, R. Buffenstein, and A. J. Hulbert, "Membrane phospholipid composition may contribute to exceptional longevity of the naked mole-rat (Heterocephalus glaber): a comparative study using shotgun lipidomics," Experimental Gerontology, vol. 42, no. 11, pp. 1053-1062, 2007.

[58] A. A. Puca, P. Andrew, V. Novelli et al., "Fatty acid profile of erythrocyte membranes as possible biomarker of longevity," Rejuvenation Research, vol. 11, no. 1, pp. 63-72, 2008. 


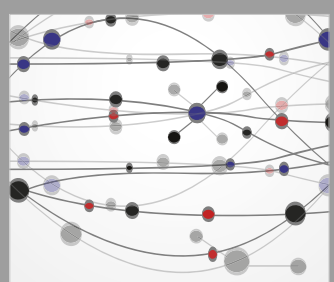

The Scientific World Journal
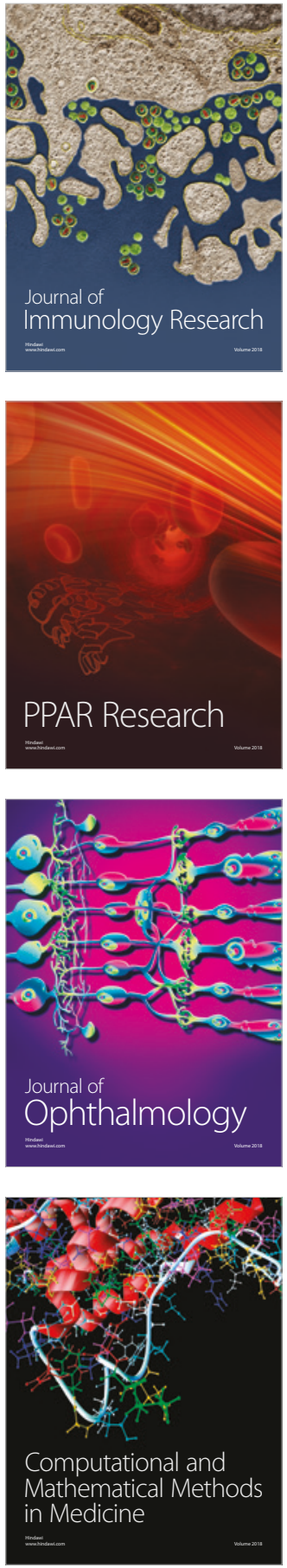

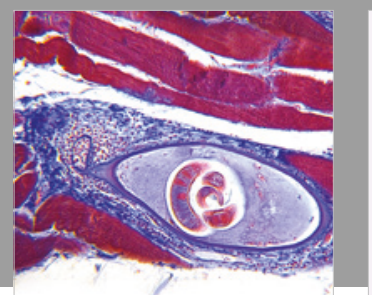

Gastroenterology Research and Practice

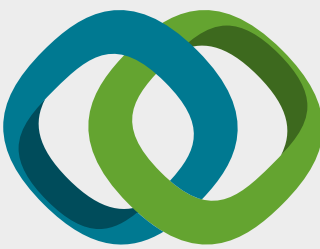

\section{Hindawi}

Submit your manuscripts at

www.hindawi.com
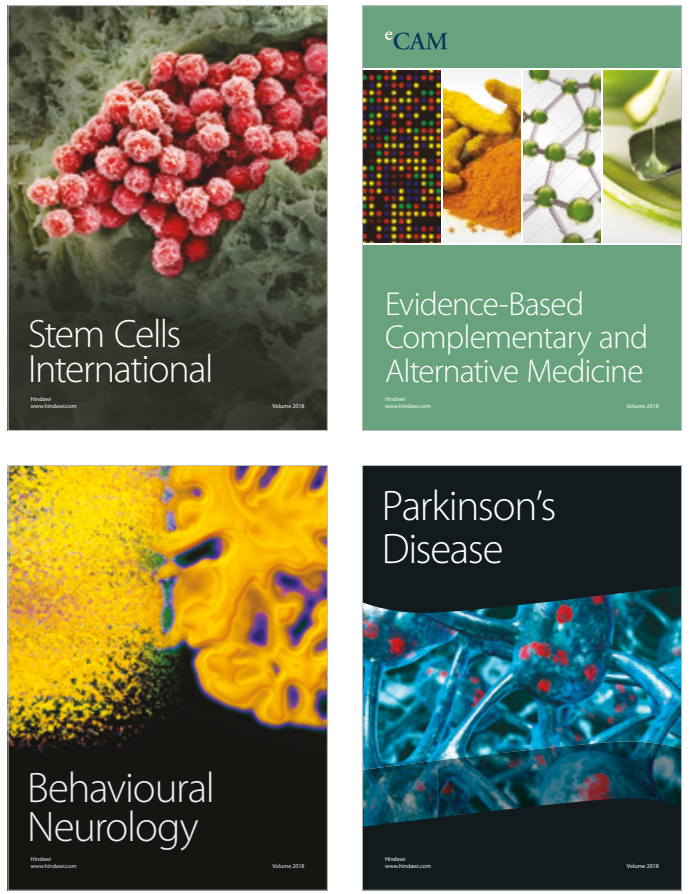

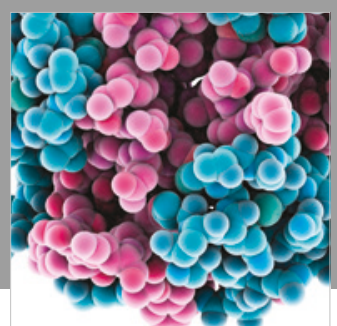

ournal of

Diabetes Research

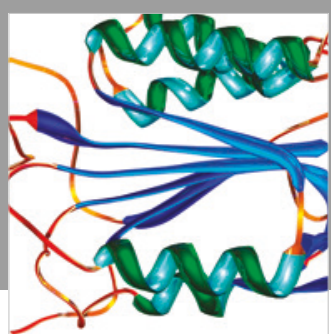

Disease Markers
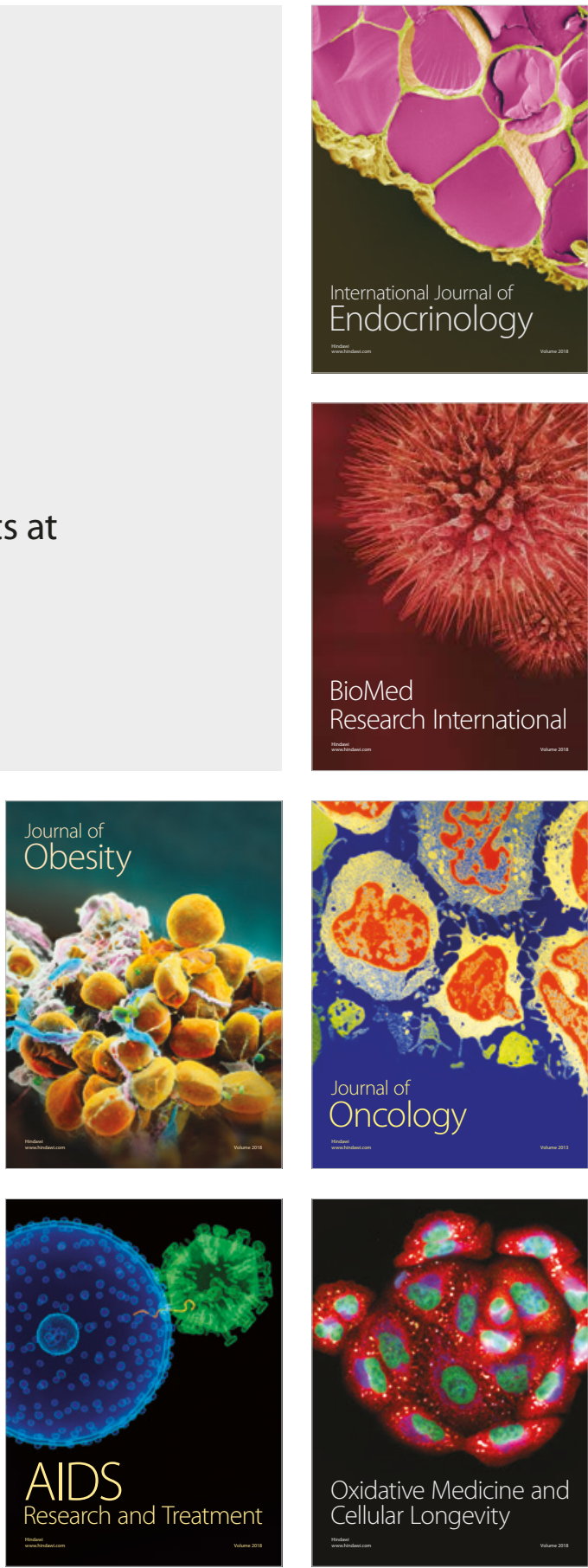